\title{
Mentoría docente para instalar estrategias de resolución de problemas en matemáticas iniciales
}

\author{
Cristian Iván Ramos Arrepol \\ Orcid: 0000-0003-3884-0278 \\ c_ramosa@inacap.cl \\ INACAP, Sede Concepción-Talcahuano \\ Carlos Alberto Acevedo Cossio \\ Orcid: 0000-0001-6504-8230 \\ carlos.acevedo17@inacapmail.cl \\ INACAP, Sede Concepción-Talcahuano
}

\section{Resumen}

El objetivo de este trabajo es presentar una instancia de innovación que se realizó en torno al trabajo colaborativo para los académicos de las asignaturas de matemática implementando los parámetros de la tutoría socioformativa para realizar una mentoría a un grupo de académicos nóveles en la institución, sobre una iniciativa de investigación y desarrollo denominado ARPA. Del total de académicos de ingreso reciente $(N=11)$, se eligieron cinco de manera aleatoria para implementar el proceso de acompañamiento, sin establecer distinciones de ningún tipo. Se realizaron reuniones de reflexión grupal periódicas y caminata de aula (acompañamiento docente), el impacto fue valorado en términos de asistencia, calificaciones y entrevistas de satisfacción. Los resultados de la intervención reflejan mejores resultados en términos de participación y rendimiento en los cursos de los profesores participantes, además de la satisfacción con la práctica e involucramiento con la institución por parte de los académicos.

Palabras clave: mentoría docente, resolución de problemas, inducción de académicos, educación superior. 


\begin{abstract}
The aim of this paper is to present an instance of innovation for the mathematics framework in the first year of higher education. It consisted on the implementación of the parameters of socio-formative tutoring to mentor a group of new academics in the institution on the ARPA method of mathematics teaching. From the total number of recently admitted academics $(N=11)$, five were randomly chosen to implement the accompaniment process, without distinctions of any kind. Regular group reflection meetings and classroom walks were held, the impact of this intervention was assessed in terms of attendance and qualifications of students and satisfaction interviews for both actors. The results of the intervention reflect an increase in terms of participation and performance in the courses of the intervened teachers, in addition of satisfaction with the practice and involvement with the institution reported by the academics.
\end{abstract}

Key words: teaching mentoring, problem solving, teacher induction, higher education.

\title{
1. Problema y justificación
}

En el marco de la mejora de la enseñanza, en cursos iniciales de matemática a nivel universitario, se capacita a docentes durante un año (2017-2018) en relación con una iniciativa de investigación y desarrollo denominado ARPA (Activando Resolución de Problemas en Aula) (Felmer y Perdomo-Díaz, 2017), que consiste en la implementación de actividades de enseñanza y aprendizajes, con eje en la resolución de problemas (RP). Esto insta a crear un modelo de docente, llamado docente experto en $R P$, quien motiva y acompaña al estudiante en la adquisición de conocimientos cuya orientación y aseguramiento del ejercicio formativo, se realiza a través de mentorías docentes. La implementación y metodología se describen en este artículo.

Los procesos que experimentan los nuevos académicos, desde que comienzan a formar parte de la Institución hasta llegar a la implementación de actividades declaradas en los planes de clases de cursos iniciales de matemática, es complejo, así como asegurar la efectividad de metodologías en el aula. La pregunta que orienta este trabajo es: «iCómo potenciar la implementación de ARPA en las asignaturas de matemáticas en primer año?». Para 
enfrentar este desafío se desarrolla un plan de acompañamiento y orientación, a través de mentorías docentes, a nivel de sede, que no solo permite armonizar y fomentar el correcto ejercicio formativo de la asignatura, sino también motivar el intercambio de experiencias en los académicos.

\section{Marco teórico}

El cambio en el acceso y los paradigmas educativos han revolucionado la concepción tradicional desarrollada sobre cómo se constituye la vida universitaria, generando la necesidad de establecer estrategias que permitan atender a la diversidad del alumnado para alcanzar los objetivos de enseñanza-aprendizaje esperados, puesto que, por medio de la asimilación y acomodación de la información, cada estudiante construye el conocimiento en un proceso orientado y recíproco con el académico (Domingo y Gómez, 2014).

Lo anterior requiere aunar conceptos, estrategias, metodologías, actividades y resultados en torno a la gestión de clases, para que se propicien actividades innovadoras que favorezcan el aprendizaje significativo de los estudiantes, pero que también respondan a la satisfacción, el compromiso y el involucramiento docente, tanto con la institución como con sus pares y sus estudiantes.

\subsection{Trabajo colaborativo}

Durante tiempo, se ha concebido el trabajo colaborativo como una estrategia de aprendizaje que está centrado en los estudiantes, que exige que ellos lo desplieguen como una competencia necesaria para su desarrollo académico y futuro laboral (Tobón, 2015); pero, si nos centramos en las concepciones clásicas de Vigotsky (1979) en relación con el aprendizaje social, esta acción también debiera verse reflejada en los docentes, es decir, no solo ha de ser un trabajo colaborativo implícito, sino que debe poseer una estructura que pueda ser evidenciada por los estudiantes, que permita el surgimiento de un tipo de aprendizaje observacional. Así, si el estudiante observa, percibe y hace consciente el modelo operante en sus docentes, dicha práctica puede ser un 
potente facilitador para adaptarse y arraigar esta estrategia en sus propios paradigmas académicos y laborales.

De acuerdo con Acevedo, Cifuentes y Espinoza (2019), el proceso educativo desde la perspectiva del docente implica un deber estar en constante aprendizaje y diálogo entre pares, que permita favorecer el capital decisorio de cada área y escuela. Así, el aprendizaje colaborativo entre pares docentes implica el afianzamiento de la organización -ecología de aprendizaje-. Ahora bien, para generar un impacto duradero en la dinámica docente, este proceso de aprendizaje entre pares, que se denominará mentorías, debe centrarse en la continua práctica reflexiva.

\subsection{Mentorías}

Una acción que apunta hacia el cambio y mejora educativa es la mentoría, entendida como un proceso, con independencia del contexto en el que se reproduzca, que es largo y profundo. Su objetivo es dirigir y guiar el autodesarrollo personal, académico y profesional del aprendiz y, se caracteriza por la confidencialidad, el compromiso ético y la promoción de una relación de confianza, basándose en la empatía y asertividad (Fernández, Belando y González, 2017).

Las complejidades de implementar una cultura orientada hacia el compromiso, la progresión y el foco en el estudiante radican en armonizar la filosofía de las propuestas y la instalación de la diversidad de diálogos y conversaciones que emergen entre los diferentes subsistemas. En este sentido, siguiendo el modelo ecológico de Urie Bronfenbrenner (Schmidt y Dos Santos, 2016), es posible ubicar en el eje de análisis al microsistema que corresponde a sala de clases, donde la interacción entre estudiante y docente constituye un espacio de construcción de aprendizaje. Luego, identificar un mesosistema que corresponde a las interacciones de la comunidad educativa en la sede, que implica no solo la participación de los estudiantes y docentes, sino también la relación y las conversaciones que existen entre estudiantesdocentes y administrativos y estudiantes-docentes y directivos. Posteriormente, en un exosistema debemos establecer decisiones a nivel administrativo y curricular que toman los directores en sede y a nivel nacional (en este caso estudiantes y docentes no 
participan de las decisiones, pero sí se ven involucrados en estas) (Acevedo y Cifuentes, 2018).

Si bien la investigación demuestra que existe controversia en cuanto a las concepciones en torno a las funciones y roles que se deben llevar a cabo en el proceso de mentoría (Orland-Barak, 2005; Malderez y Bodóczky, 1999; Flóres, 2004; Shaw, 1992), Randall y Thornton (2001, como se citó en Díaz y Bastías, 2013) destacan que los mentores no solo tienen un rol profesional de desarrollo, sino también un rol institucional y una función evaluadora. De esta manera, el mentor puede desempeñar una amplia variedad de roles y funciones, desde los roles técnicos y evaluativos hasta roles más personales y de desarrollo.

\section{Método de investigación}

La metodología de base es la tutoría socioformativa (López y Tobón-Tobón, 2017), que implica una serie de seis ejes en la intervención, que van desde la identificación de los actores hasta la ejecución de la implementación, junto con el seguimiento del desempeño del académico, a lo largo del primer semestre académico del año 2019 (marzo-junio). La intervención fue realizada sobre académicos noveles en la institución; del total de recién ingresados $(\mathrm{N}=11)$ se eligieron cinco académicos al azar para asegurar la abordabilidad del trabajo a desarrollar.

Las herramientas utilizadas para la valoración de la intervención consistieron en la comparación del promedio de las primeras cinco evaluaciones considerando que, de acuerdo con el diseño instruccional de la asignatura, todos los docentes utilizan los mismos instrumentos, con objetivos de aprendizajes iguales, aplicadas los mismos días con ítems distintos por docente, situación que cambia en la sexta y séptima al tratarse de evaluaciones del contenido en relación con el área en que se dicta la clase. Además, se revisan los promedios de reprobación temprana (calificación e inasistencia) y se registran impresiones de académicos y estudiantes ante el proceso de instalación de la estrategia formativa.

El esfuerzo descrito se plantea como posible respuesta a las necesidades del Proyecto Educativo Institucional, basado en la formación de competencias, para instalar este método como una 
opción viable a pesar de las dificultades que representan estas iniciativas (Díaz, 2005; Villarroel y Bruna, 2014; INACAP, 2015). A continuación, se describen las principales etapas del presente trabajo.

\subsection{El perfil de entrada del docente}

Sobre la base de las necesidades detectadas de las direcciones de carreras (escuelas) en que los académicos prestan servicios, el método de enseñanza-aprendizaje establecido, las distintas experiencias a nivel nacional y según el documento institucional guía Cómo enseñamos matemática en INACAP (institución académica chilena donde se desarrolló la investigación), se estableció el perfil de entrada, con las siguientes características:

- Potenciador de la habilidad de resolución de problemas: el docente demuestra fomentar la habilidad de argumentación en el alumno que le permite adquirir nuevas estrategias para resolver problemas y trabajar en equipo, facilitando relacionarse con pares, dándole la oportunidad de explorar nuevas situaciones de aprendizaje y protagonismo en su aprendizaje.

- Comprometido con el trabajo bien hecho: el docente conoce y aplica los lineamientos de los programas de estudio de asignaturas de ciencias básicas, participa de jornadas de planificación aportando en la generación e implementación de estos, comparte experiencias, socializando buenas prácticas entre pares en instancias de observaciones de clases y organizado en los requerimientos administrativos, que evidencien los procesos y procedimientos que la institución exija.

- Innovador en docencia: el docente tiene la motivación de un desarrollo profesional docente continuo, con iniciativa de participar en instancias de capacitación, exposición e investigación relacionadas a la educación matemática (congresos, grupos de innovación y/o seminarios), con lo que proyecta estas actitudes a sus estudiantes.

\subsection{Reclutamiento docente}

Para el semestre iniciado en otoño de 2019 se fijan secciones de cursos de matemática iniciales para intervenir. Con esto, se ini- 
cia el procedimiento de reclutamiento y selección de profesores donde se recibieron 22 currículos. El análisis de estos muestra 18 docentes habilitados para la siguiente fase de entrevista personal, etapa en la que 15 docentes siguen en carrera. Luego, los postulantes realizan una microclase, con la presencia de evaluadores (jefes de carrera, asesor pedagógico y coordinador de asignaturas de ciencias básicas), quienes participan en la clase simulada, recreando el comportamiento de alumnos de primer año, con sus dificultades e inquietudes. Ahí se analizan las habilidades del docente, según el perfil de ingreso, un proceso que habilitó a 11 nuevos docentes para ser contratados.

\subsection{La caminata de aula}

Denominamos caminata de aula al acompañamiento en la formación de docentes, desde la observación y reflexión de buenas prácticas en aula con docentes expertos en RP.

\subsubsection{El primer paso}

La primera actividad, antes del inicio del semestre, es una inducción de la metodología ARPA a los nuevos docentes, con dos momentos importantes. Primero, resuelven una actividad de aprendizaje experimentando como alumnos estrategias que docentes expertos en RP les aplican, para después reflexionar de lo realizado. Segundo, deben planificar una actividad para la primera clase de sus respectivos cursos.

Luego, en la segunda semana del semestre, se observan las clases de los docentes, según la pauta de observación de ARPA, que mide distintas actividades que debe realizar el docente en el aula, para luego conversar con este, reflexionando acerca de aciertos, dificultades, fortalezas y lo que él o ella debería mejorar durante su clase. Finalmente, se le plantea el desafío de que «él o ella sea el observador de aula» y se le designa a un docente experto en RP como observado.

Esta primera etapa se cierra con una reunión de reflexión con todos los participantes, en la cual se analiza en forma grupal e individual las actividades y acciones observadas y se plantean plazos y desafíos a realizar durante el semestre. 


\subsubsection{Segunda ronda}

En la semana siete del semestre se realiza la segunda ronda de observaciones en donde se comparan los comentarios hechos del primer acompañamiento midiendo los avances en la aplicación de la estrategia, junto con esto se verifica el cumplimiento de los desafíos adquiridos de cada docente que participa de esta iniciativa.

Luego, ellos deben compartir sus buenas prácticas mediante la observación de clases entre pares, tomando cada uno el papel de observador y observado y entregando un informe con las anotaciones y comentarios del acompañamiento a la coordinación de ciencias básicas.

Para finalizar, nuevamente se realiza una reunión de socialización, donde se reflexiona acerca de la experiencia de esta segunda etapa y del proceso en general.

\subsubsection{Ronda final}

La última instancia de observaciones pretende cerrar en la semana 15 midiendo si el docente ha adquirido las principales competencias que le permitan seguir en la aplicación de la metodología $\mathrm{RP}$ en los cursos siguientes. A esto se le suma una reunión final, donde se exponen resultados y objetivos alcanzados durante el semestre.

\subsection{Abordaje de situaciones críticas}

A partir de las eventuales situaciones críticas emergentes (descontento de estudiantes con las clases) que conlleva la aplicación de nuevos procesos es como se establece un protocolo para solucionarlas. Se trabaja con docentes nuevos o antiguos, según se requiera, en un afán de alinear criterios según descriptores y plan de clases de las asignaturas intervenidas.

1. El tutor identifica un curso que ha recibido reclamos o acotaciones de los alumnos, o que desde el punto de vista del docente, este no se alinea con los alumnos.

2. Con la participación del director de carrera y/o coordinador se conversa con los estudiantes, donde se toma nota de la visión de ellos.

3. El coordinador de ciencias básicas entrevista al académico, informando de las inquietudes de los estudiantes. Luego, obser- 
va su clase, retroalimentando según las directrices del perfil de docente en RP, y adquieren acuerdos para el semestre.

4. Se aplican las medidas de mejora, para lo cual el profesor tiene la opción de observar una clase de docente experto en RP, para luego llevar esto al aula. Finalmente, en una charla final se sacan conclusiones con él. Posteriormente, en una reunión con los alumnos, se testean los cambios vistos por ellos desde el punto de vista académico.

\section{Resultados}

Los cinco docentes elegidos en el presente trabajo participaron el cien por cien de las reuniones de reflexión. Además, se designa un docente antiguo para implementación de dificultades emergentes. A continuación, en la tabla 1 se presentan algunas reflexiones realizadas por los docentes observados durante las reuniones periódicas a las cuales debían asistir.

Tabla 1. Aciertos y dificultades de docentes en la aplicación de la metodología.

\begin{tabular}{ll}
\hline \multicolumn{1}{c}{ Aciertos } & \multicolumn{1}{c}{ Dificultades } \\
\hline $\begin{array}{l}\text { Validación de las estrategias de los estu- } \\
\text { diantes ante la resolución de problemas. }\end{array}$ & $\begin{array}{l}\text { El uso frecuente del celular por parte de los } \\
\text { alumnos. }\end{array}$ \\
\hline $\begin{array}{l}\text { El diseño instruccional permite estandarizar } \\
\text { actividades. }\end{array}$ & $\begin{array}{l}\text { Tengo, como docente, tendencia a enseñar } \\
\text { métodos propios en vez de estimular el des- } \\
\begin{array}{l}\text { Hacer pasar a los estudiantes a la pizarra } \\
\text { promueve el compartir estrategias de reso- } \\
\text { lución entre pares. }\end{array}\end{array}$ \\
$\begin{array}{ll}\text { cubrimiento de los alumnos. } \\
\begin{array}{l}\text { Permite el razonamiento matemático desde } \\
\text { la interacción en lugar de la repetición. }\end{array}\end{array}$ & $\begin{array}{l}\text { Falta interactuar con asignaturas de espe- } \\
\text { cialidad para que la enseñanza sea signifi- } \\
\text { cativa. }\end{array}$ \\
\hline $\begin{array}{l}\text { RP-ARPA constituye un acierto, ya que los } \\
\text { cursos que han mostrado mayor capacidad } \\
\text { de reflexión matemática, se ha desarrollado } \\
\text { un trabajo más colaborativo entre pares. }\end{array}$ & $\begin{array}{l}\text { Los alumnos piden explicación del profesor, } \\
\text { de materias que no se acuerdan o no vieron } \\
\text { en la enseñanza media. }\end{array}$ \\
\hline
\end{tabular}

Los académicos valoran la implementación de estrategias que homogeneicen el proceso de enseñanza entre los distintos cur- 
sos y reconocen que esto potencia las prácticas docentes, pues permite reflexionar conjuntamente sobre los resultados de la implementación; destaca el efecto que tiene sobre el estudiante en términos de potenciar su participación para desarrollar aprendizajes en los que el rol del docente se sitúa desde la guía. Así, también se puede observar la dificultad que representa para el académico movilizar tanto al estudiante como sus propios recursos hacia un cambio de paradigma que rompe la inercia y hace partícipe al aprendiz, pues se requiere de ambos para llevar a cabo el proceso.

Los profesores también reflexionan sobre sus fortalezas en forma grupal y adquieren individualmente compromisos. Las fortalezas se observan relacionadas principalmente con elementos vocacionales y actitudinales hacia el trabajo en equipo, mientras que el compromiso apunta específicamente a la mejora continua, como se presenta en la tabla 2.

Tabla 2. Fortalezas y compromisos de docentes observados.

\begin{tabular}{ll}
\hline \multicolumn{1}{c}{ Mis fortalezas } & \multicolumn{1}{c}{ Me comprometo } \\
\hline Capacidad de adaptarme a un grupo. & Seguir mejorando. \\
\hline Empático con estudiantes. & $\begin{array}{l}\text { A participar activamente en actividades de } \\
\text { coordinación con otros docentes. }\end{array}$ \\
\hline $\begin{array}{l}\text { Buena disposición a recibir observaciones } \\
\text { y comentarios. }\end{array}$ & $\begin{array}{l}\text { A organizar los tiempos en clases para for- } \\
\text { malizar los contenidos. }\end{array}$ \\
\hline Capacidad de adaptarme a un grupo. & Seguir mejorando. \\
\hline $\begin{array}{l}\text { Pasión por las matemáticas y la capacidad } \\
\text { de enseñar en un lenguaje cotidiano. }\end{array}$ & $\begin{array}{l}\text { A organizar a trabajar colaborativo en la } \\
\text { planificación de contenidos y evaluaciones. }\end{array}$ \\
\hline
\end{tabular}

La medición según las cinco primeras evaluaciones, además, de la estandarización de contenidos, también se considera que estas equivalen, en porcentaje, al $65 \%$ de la nota final, y desde experiencias de semestres anteriores, los resultados no serán muy alejados de la realidad. Se observa en el gráfico 1 que los alumnos con docentes acompañados obtienen un 4,2 de promedio, mientras que sin acompañamiento es de 3,6. 
Gráfico 1. Comparación de promedio de notas de alumnos.

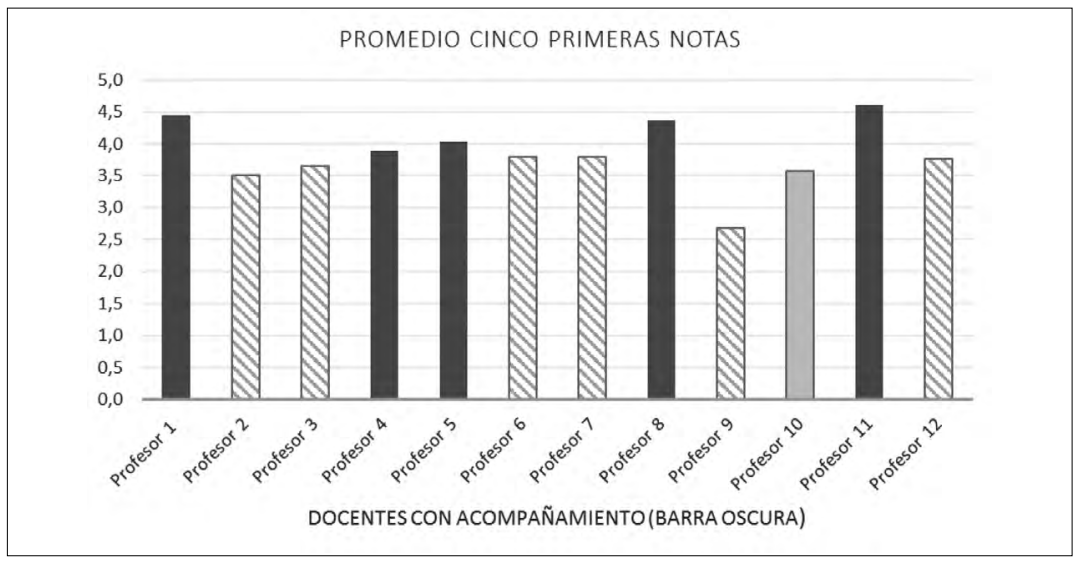

Fuente: Elaboración propia (2019).

Así, a continuación (gráfico 2), se muestra la comparación de porcentaje de alumnos con aprobación, de docentes acompañados y no acompañados. También la estadística de un docente apoyado en situación crítica emergente (Profesor 10). De promedio, el $59 \%$ de los alumnos con docentes acompañados aprueban; en cambio, en el caso de los que están sin acompañamiento, se desciende hasta el $44 \%$.

Gráfico 2. Comparación de porcentajes de alumnos aprobados.

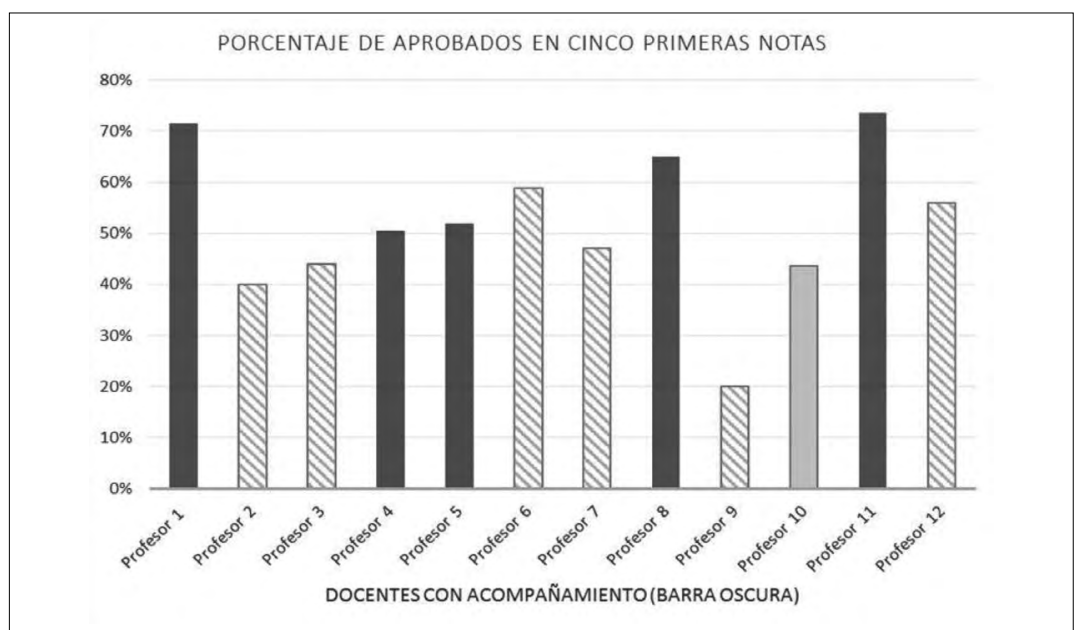

Fuente: Elaboración propia (2019). 
Uno de los requisitos para aprobar el curso es que la asistencia mínima a clases de alumnos sea de un $60 \%$; por tanto, si el alumno, en la fecha de la medición (semana 15), tiene un porcentaje inferior al $40 \%$, está reprobado por inasistencia (RI). A continuación (gráfico 3), se muestra este porcentaje para cada profesor en estudio. De promedio los docentes acompañados tienen un $13 \%$ de alumnos en esta situación, versus el $22 \%$ de los que no.

Gráfico 3. Comparación porcentaje de estudiantes reprobados por inasistencia.

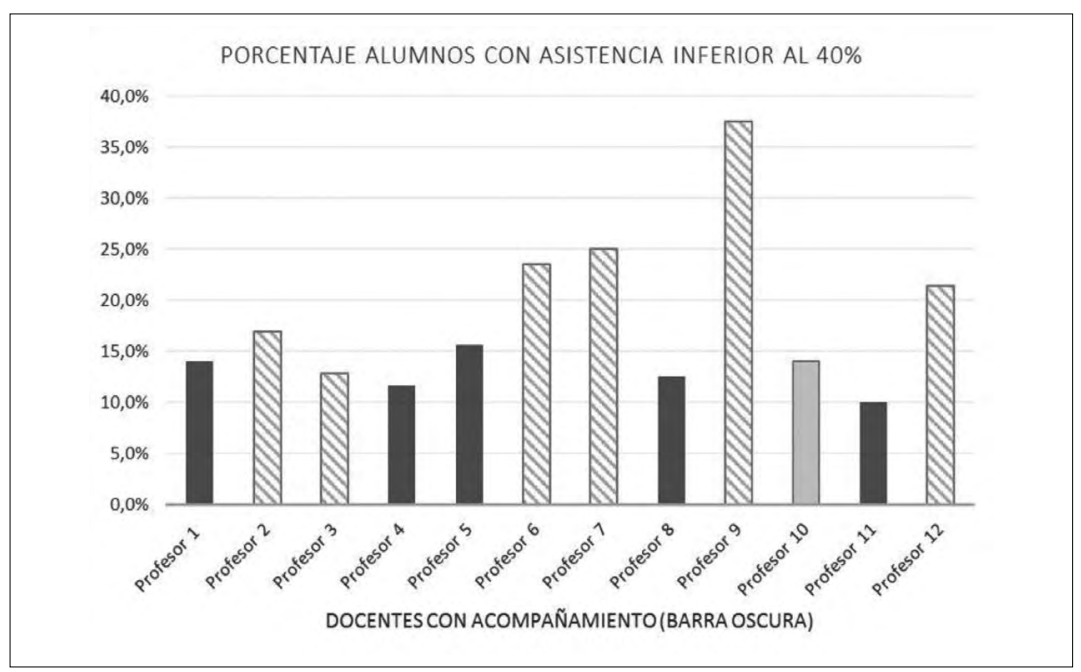

Fuente: Elaboración propia (2019).

De los estudiantes se recopiló la información, de forma anónima y voluntaria, en cuanto a visitas a los cursos (semana 10), sobre qué opinan de la metodología. En la tabla 3 se muestran las principales ideas.

Se observa que los estudiantes valoran el empleo de nuevas estrategias, sin embargo, cuesta adaptarse a la nueva forma de entregar los conocimientos y además, sienten que los docentes no están haciendo clases; sin embargo, reconocen la mejoría existente de la metodología nueva, ya que ahora son ellos que tienen que descubrir cómo se resuelven los problemas. 
Tabla 3. Opinión de alumnos sobre metodología aplicada por docentes en estudio.

\begin{tabular}{|c|c|}
\hline \multicolumn{2}{|c|}{ Referente a la metodología, como alumno mi opinión es: } \\
\hline $\begin{array}{l}\text { Nos permite conocer e intercambiar ideas con } \\
\text { nuestros compañeros. }\end{array}$ & $\begin{array}{l}\text { Poco tiempo para el hacer los ejercicios en } \\
\text { clases. Siento que el docente no pasa materia }\end{array}$ \\
\hline $\begin{array}{l}\text { El profesor nos ayuda a desarrollar la argu- } \\
\text { mentación de la solución. }\end{array}$ & $\begin{array}{l}\text { en clases. } \\
\text { Las guías no se hacen completas en clases. }\end{array}$ \\
\hline $\begin{array}{l}\text { El trabajo y apoyo fuera de clases es muy } \\
\text { bueno. }\end{array}$ & $\begin{array}{l}\text { Solo resolvemos un problema en la clase. } \\
\text { El docente trabaja en clases con los mismos }\end{array}$ \\
\hline $\begin{array}{l}\text { El docente nos hace comparar estrategias y } \\
\text { Ilegar a una solución en común. }\end{array}$ & $\begin{array}{l}\text { ejercicios y en el momento del certamen los } \\
\text { cambia. }\end{array}$ \\
\hline $\begin{array}{l}\text { El profesor cuando nos ayuda en clases se } \\
\text { comunica con todo el grupo y hace que nos }\end{array}$ & $\begin{array}{l}\text { El docente nunca da la respuesta a los ejer- } \\
\text { cicios. }\end{array}$ \\
\hline $\begin{array}{l}\text { conozcamos. } \\
\text { El trabajo en }\end{array}$ & $\begin{array}{l}\text { El docente siempre trata de que apliquemos lo } \\
\text { que sabemos de la enseñanza media. }\end{array}$ \\
\hline $\begin{array}{l}\text { aprendemos en la sala. Es entretenido formar } \\
\text { grupos siempre distintos de trabajo. }\end{array}$ & $\begin{array}{l}\text { El académico no hace clases solo nos hace } \\
\text { resolver la guia en la clase. }\end{array}$ \\
\hline $\begin{array}{l}\text { La clase es más dinámica y siempre hay algo } \\
\text { nuevo que hacer y nos ayuda a exponer en }\end{array}$ & $\begin{array}{l}\text { Se utilizan los mismos ejemplos en clases y el } \\
\text { docente en el certamen pregunta otra cosa. }\end{array}$ \\
\hline $\begin{array}{l}\text { público. } \\
\text { El trabajo en grupo hace que nos conozcamos } \\
\text { mejor. Siempre el docente nos motiva a seguir } \\
\text { descubriendo soluciones nuevas. }\end{array}$ & $\begin{array}{l}\text { Un método de enseñanza que tengo que me } \\
\text { hace descubrir la materia que docente quiere } \\
\text { ver en clases. Para esta metodología nueva, } \\
\text { me falta tiempo para asimilarla mejor. }\end{array}$ \\
\hline $\begin{array}{l}\text { Se genera discusiones con otros grupos de } \\
\text { trabajo. }\end{array}$ & $\begin{array}{l}\text { No alcanza a desarrollar todas las guias, y yo } \\
\text { debo buscar las fórmulas. }\end{array}$ \\
\hline $\begin{array}{l}\text { Ayudar a compañeros sirve para entender me- } \\
\text { jor los problemas y la clase es más entretenida } \\
\text { que antes. }\end{array}$ & \\
\hline
\end{tabular}

\section{Conclusiones y discusión}

El proceso de mentoría resulta positivo para la implementación de métodos de enseñanza por parte de académicos que recién se integran a la cultura de una institución. Permite abordar las inquietudes y orientar el proceso de manera tal que permite al docente transmitir a sus estudiantes una percepción de dominio que potencia el involucramiento y facilita la superación de las dificultades naturales que toda instalación de metodologías exige (Díaz, 2005; Villarroel y Bruna, 2014). Aunque las limitaciones de este trabajo consisten en la reducida muestra y, por eso mismo, no implica un análisis estadístico más profundo, los 
autores esperan que sirva como semillero para nuevas iniciativas que profundicen en la estrategia y que realcen la importancia de realizar un acompañamiento continuo a los profesores nóveles, basados en la utilidad que tiene para el estudiante y los beneficios que genera a los académicos y la institución, el trabajo colaborativo de estos orientados por los docentes con mayor experiencia en resolución de problemas, que organicen la experiencia formativa desde una perspectiva reflexiva para dotar de sentido la labor docente.

\section{Referencias bibliográficas}

Acevedo, C.; Cifuentes, C. (2018). «Engagement: Intervención Sistémica para el Desarrollo del Involucramiento Académico en Primer Año». Congresos CLABES. Recuperado de: <https://revistas.utp.ac.pa/ index.php/clabes/article/view/2010>.

Acevedo, C.; Cifuentes, C.; Espinoza, M. (2019). Análisis institucional a nivel sede sobre el Involucramiento Académico Estudiantil y Docente. Santiago: INACAP.

Díaz, M. (2005). Modalidades de enseñanza centradas en el desarrollo de competencias. Orientaciones para promover el cambio metodológico en el espacio europeo de educación superior. España: Universidad de Oviedo.

Díaz, C.; Bastías, C. (2013). «Los procesos de mentoría en la formación inicial docente». Revista Internacional de Investigación en Ciencias Sociales, 9(2): 301-315. Recuperado de: <https://dialnet.unirioja.es/ servlet/articulo?codigo $=4714183>$.

Domingo, A.; Gómez, M. (2014). La práctica reflexiva. Bases, modelos e instrumentos. Madrid: Narcea.

Felmer, P.; Perdomo-Díaz, J. (2017). «Un programa de desarrollo profesional docente para un currículo de matemática centrado en las habilidades: la resolución de problemas como eje articulador». Educación matemática, 29(1): 201-217. DOI: <https://dx.doi.org/10.24844/ em2901.08>.

Fernández, C.; Belando, M.; González, M. (2017). «Mentoría pedagógica para profesorado universitario novel: estado de la cuestión y análisis de buenas prácticas». Estudios sobre educación, 33: 49-75.

Flóres, R. (2004). Evaluación pedagógica y cognición. Bogotá: McGraw Hill. INACAP (2015). Modelo Educativo Institucional. Recuperado de: <http:// www.inacap.cl/web/acerca-de/Modelo-Educativo-2015.pdf>. 
López, V. H.; Tobón-Tobón, S. (2017). «La tutoría socioformativa en la educación superior». Revista Docencia e Investigación, 1(27): 33-58. Recuperado de: <http://hdl.handle.net/10578/19042>.

Malderez, A.; Bodóczky, C. (1999). Mentor courses. Cambridge: Cambridge University Press.

Orland-Barak, L. (2005). «Lost in translation: mentors learning to participate in competing discourses of practice». Journal of Teacher Education, 56(4): 66-79.

Shaw, R. (1992). Teacher training in secondary schools. Londres: Kogan Page.

Tobón, S. (2015). Estrategias para fortalecer la tutoría desde la socioformación. México: CIFE.

Villarroel, V.; Bruna, D. (2014). «Reflexiones en torno a las competencias genéricas en educación superior: Un desafío pendiente». Psicoperspectivas, 13(1): 22-34. DOI: <http://dx.doi.org/10.5027/psicoperspectivas-Vol13-Issue1-fulltext-335>. 

1. La innovación educativa en el contexto de la

Educación Superior Técnico-Profesional.

Claudio Maregatti Solano, María luisa Arancibia Muñoz, Rosa Eliana Romero Alonso

1. Introducción

2. La transformación de las instituciones de

Educación Superior Técnico-Profesional

3. De las prácticas docentes al saber académico

4. El aporte al conocimiento científico sobre la

Educación Superior Técnico-Profesional . . . . . . . . . 15

5. Referencias bibliográficas .................. . 21

2. Caracterización de estudiantes de Educación Técnico-Profesional respecto a su Educación Secundaria: reflexiones para la Educación Superior

Javier Torres-Vallejos, Juan Ignacio Venegas Muggli, Simón Mundaca Toledo, Juan Carlos Oyanedel Sepúlveda

1. Introducción . . . . . . . . . . . . . . . . . . . 26

2. Metodología ......................... . 30

2.1 Muestra . . . . . . . . . . . . . . . . . . . . . 30

2.2 Variables . . . . . . . . . . . . . . . . . . . . . 32

2.3 Tratamiento de bases de datos . . . . . . . . . . . . 32

2.4 Análisis de datos..................... 33

3. Resultados ........................... 33

3.1 Características educativas y académicas en

Educación Superior ..................... . 34

3.2 Características educativas y académicas del establecimiento secundario de egreso de Enseñanza Media . . . . . . . . . . . . . . . . . . . . 36

4. Discusión y conclusiones. . . . . . . . . . . . . . . 37

5. Referencias bibliográficas ................. . . 39 
3. Aportes hacia la construcción de un perfil docente para el primer año: la importancia del vínculo empático, el valor académico y el trabajo colaborativo

Carlos Alberto Acevedo Cossio

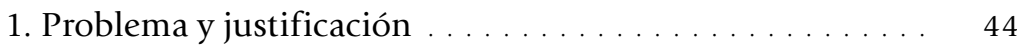

2. Marco teórico ....................... 45

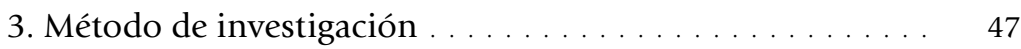

3.1 Diseño. . . . . . . . . . . . . . . . . . . 47

3.2 Participantes...................... 47

3.3 Técnicas e instrumentos de recolección de datos. . . . 48

3.4 Análisis de datos. . . . . . . . . . . . . . . . . . . . . 48

4. Resultados ... . . . . . . . . . . . . . . . . . . . . . . 49

4.1 Análisis de las entrevistas realizadas . . . . . . . . . 49

4.2 Análisis de cuestionarios . . . . . . . . . . . . . 50

4.2.1 Cuestionario de Estilo y Eficacia del Liderazgo . . 50

4.2.2 Cuestionario de Involucramiento Académico . . . 51

4.2.3 Índice de Reactividad Interpersonal . . . . . . . . . 52

5. Conclusiones y discusión ................. . 53

6. Referencias bibliográficas .................. 55

4. Mentoría docente para instalar estrategias de resolución de problemas en matemáticas iniciales

Cristian Iván Ramos Arrepol, Carlos Alberto Acevedo

Cossio

1. Problema y justificación . . . . . . . . . . . . . . . . 58

2. Marco teórico . . . . . . . . . . . . . . . . . . . . . . . 59

2.1 Trabajo colaborativo . . . . . . . . . . . . . . . . . 59

2.2 Mentorías ......................... 60

3. Método de investigación .................... 61

3.1 El perfil de entrada del docente . . . . . . . . . . . 62

3.2 Reclutamiento docente. . . . . . . . . . . . . 62

3.3 La caminata de aula . . . . . . . . . . . . . . . . . . 63

3.3.1 El primer paso . . . . . . . . . . . . . . . . 63

3.3 .2 Segunda ronda . . . . . . . . . . . . . . . . . . 64

3.3.3 Ronda final. . . . . . . . . . . . . . . . . . 64

3.4 Abordaje de situaciones críticas . . . . . . . . . . . . 64

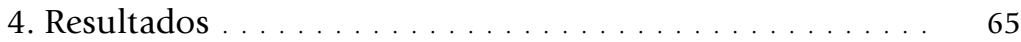

5. Conclusiones y discusión. . . . . . . . . . . . . . . . 69

6. Referencias bibliográficas ................... 70 
5. Clase invertida para la formación inicial de educadoras diferenciales sobre aprendizaje matemático

Laura Marjorie Espinoza Pastén

1. Problema y justificación . . . . . . . . . . . . . . . . . . 74

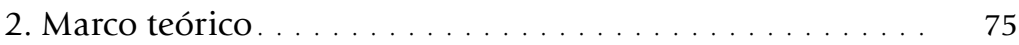

3. Proceso de la innovación. . . . . . . . . . . . . . . 76

3.1 Contexto ........................ 76

3.2 Diseño didáctico . . . . . . . . . . . . . . . 77

4. Resultados ......................... . 80

4.1 Resultados considerando el rendimiento académico. . 80

4.2 Resultados considerando la valoración de las estudiantes $\quad 81$

5. Discusión y conclusiones . . . . . . . . . . . . . . . 83

6. Referencias bibliográficas ................. 84

6. ¿Cómo medir el aprendizaje en innovación? Análisis factorial confirmatorio del Innovator's Behavior Questionnaire (i)BQ en universitarios chilenos. . . . . . 87 Jorge Maluenda Albornoz, Pedro Lledó Aninat

1. Problema y justificación . . . . . . . . . . . . . . . . . . . . . 89

2. Marco teórico . . . . . . . . . . . . . . . . . . . . . 90

3. Método............................. 92

4. Resultados ......................... 93

5. Conclusiones y discusión. . . . . . . . . . . . . . . . . 96

6. Referencias bibliográficas ................. 98

7. Programa de inclusión para actores de Educación Superior Técnico-Profesional . . . . . . . . . . . . . . . . 101

Marcela Pérez Poquet, Paulina Muñoz Villalobos, Victoria Aravena Rivas

1. Problema y justificación del estudio . . . . . . . . . . . . 102

2. Marco teórico . . . . . . . . . . . . . . . . . . . . . . 105

3. Metodología de investigación ................ 108

3.1 Descripción del contexto . . . . . . . . . . . . . . . 108

3.2 Participantes. . . . . . . . . . . . . . . . . . . . 109

3.3 Instrumentos y procedimientos . . . . . . . . . . . . . . 109

4. Resultados . . . . . . . . . . . . . . . . . . . . . . . 111

5. Conclusiones y discusión. . . . . . . . . . . . . . . . . . 113

6. Referencias bibliográficas ................. 113

Los autores. . . . . . . . . . . . . . . . . . . . . . . . . ${ }^{115}$ 